\title{
Printing and characterizing plasmonic nanoparticles
}

\author{
Narcis Fosso ${ }^{1}$, David Palma de Barros ${ }^{2}$, Jane Krähenbühl ${ }^{1}$, Silvia Schintke ${ }^{1, *}$ \\ ${ }^{1}$ Laboratory of Applied NanoSciences (COMATEC-LANS), Department of Industrial Technologies, HEIG-VD, \\ University of Applied Sciences Western Switzerland (HES-SO), CH 1401 Yverdon-les-Bains, Switzerland \\ ${ }^{2}$ Ecole de Physique Appliquée, INSA, F-31077 Toulouse, France \\ Tel: (41) 2455761 67, Fax: (41) 2455776 01, e-mail: silvia.schintke@heig-vd.ch
}

\begin{abstract}
Printing plasmonic nanoparticles is of interest e.g. in the fields of large area printing for photovoltaic applications, biomedical and photonic sensor developments, as well as for digital printing of security tags for smart packaging and anti-counterfeit applications. We have studied plasmonic gold nanoparticles embedded in printable PEDOT:PSS (poly(3,4-ethylenedioxythiophene)-poly(styrenesulfonate), a transparent hole conducting organic semiconductor material). Aqueous dispersions of chemically stabilized gold nanoparticles (e.g. $40 \mathrm{~nm}$ in diameter) were used as plasmonic nanoparticle additives for the nanocomposite coating materials and inks. Inkjet printing and spincoating experiments have been performed on glass substrates.

The inkjet printed nanocomposite microstructures and thin film coatings were investigated by true non-contact atomic force microscopy (AFM), 3D profilometry, optical microscopy. Absorbance spectroscopy was used to characterize the gold nanoparticle dispersions upon synthesis and encapsulation in comparison to commercial gold nanoparticles. AFM topography and phase contrast data reveal the domain structure of PEDOT:PSS, and indicate embedding of the nanoparticles within the transparent conducting polymer printed structures. Successive printing of the PEDOT:PSS ink and gold nanoparticle dispersions leads to the contrary to significant topographic contrasts in AFM and optical profilometry. Using the coffee stain effect, we generate inkjet printed plasmonic nanocomposite microstructures that are of potential interest for the application field of smart electrically conducting and plasmonic security tags.
\end{abstract}

Keywords: plasmonics, nanoparticle inks, nanocomposites, transparent conductor, atomic force microscopy, inkjet printing

\section{INTRODUCTION}

Materials for printing transparent electrodes and plasmonic structures are of interest for transparent electrical circuits with additional optical features, e.g. for applications as smart security tags that are electrically conducting while exhibiting also characteristic optical features. Further interest is in the field of photovoltaics and flexible or printed organic electronics, and for transparent optoelectronic devices and circuits [1]. Widely studied plasmonic particles are gold nanoparticles with a characteristic size dependent optical absorbance peak in the visible range of the optical spectrum [2]. An inkjet printable transparent conducting material is the polymer poly $(3,4-$ ethylenedioxythiophene): polystyrene sulfonate (PEDOT:PSS), a hole transport material, widely used in organic electronic devices, that has been broadly studied as a candidate to replace indium tin oxide (ITO) in flat panel display and optoelectronic devices [3,4]. It's thin film conductivity can be improve significantly by using e.g. secondary dopants such as dimethyl sulfoxide (DMSO) [5,6] and/or graphene oxide [7].

In this paper, we have combined PEDOT:PSS and gold nanoparticles for microstructure inkjet printing aiming at the printing of smart nanocomposite structures for security tag applications providing electrical conductivity and optical features from plasmonic nanoparticles at once.

For successive printing of plasmonic gold nanoparticles on PEDOT:PSS, and when making use of the coffee stain effect, we fabricate topographic nano-and microstructures that are promising for the development of smart security tags that are electrically conducting and exhibit additional plasmonic features.

\section{EXPERIMENTAL}

Printing of PEDOT:PSS - gold nano-and microstructure features has been performed using a sonoplot microplotter using a glass capillary nozzle width of typically $60 \mu \mathrm{m}$ inner diameter.

\subsection{Sample preparation}

Glass slides (25 mm x $25 \mathrm{~mm}$ x $1.1 \mathrm{~mm}$, Sigma Aldrich) are used as substrates. PEDOT:PSS (1.3\%wt water dissolved, Sigma Aldrich) is used as organic transparent conducting material for thin film depositions. Inkjet printable PEDOT:PSS $(0.8 \% \mathrm{wt}$ water dissolved, Sigma Aldrich) is used for microstructure printing. Au nanoparticle dispersion (nominal diameter $40 \mathrm{~nm}$ ) are purchased from nanoComposix (40 nm Gold Nanospheres citrate stabilized, TEM diameter $42 \pm 5 \mathrm{~nm}$ and $40 \mathrm{~nm}$ PEG NanoXact ${ }^{\text {TM }}$ Gold, TEM diameter $41.5 \mathrm{~nm} \pm 3.5 \mathrm{~nm}$ ) and are also synthesized according to the Turkevic method (see below). 
Thin PEDOT:PSS/glass films of controllable thickness and various Au nanoparticle additive concentration are obtained by spin coating ( $2000 \mathrm{rpm}$ for $30 \mathrm{~s}$ ). The deposited thin films were thermally cured during $10 \mathrm{~min}$ in an oven at $160^{\circ} \mathrm{C}$. Inkjet printed structures were thermally cured during $10 \mathrm{~min}$ on a hot plate at $130^{\circ} \mathrm{C}$.

\subsection{Gold nanoparticle synthesis}

Gold nanoparticles of controllable size are synthesized following the method of Turkevich [8,2]. $5 \mathrm{~mL}$ chloric acid (Sigma Aldrich, $2 \mathrm{mg} / \mathrm{mL}$ ) are diluted in $100 \mathrm{~mL}$ distilled water and brought to $90^{\circ} \mathrm{C}$, magnetically stirred at $300 \mathrm{rpm} .1 \mathrm{~mL}$ sodium tri-citrate at $10 \mathrm{mg} / \mathrm{mL}$ are added to the solution, which is heated under agitation during 10 minutes, cooled down to room temperature before direct further use or intermediate storage in the fridge at $4^{\circ} \mathrm{C}$. For embedding gold nanoparticles into PEDOT:PSS, the nanoparticles solution is concentrated by centrifugation (9200 rpm, 15 minutes, 3 times) in three successive steps. $20 \mu \mathrm{L}$ concentrated solution are so obtained from a 5 $\mathrm{mL}$ synthesized nanoparticle solution.

\subsection{PEG encapsulation of gold nanoparticles for their embedding in PEDOT:PSS}

Upon synthesis the Au nanoparticles are electrostatically stabilized by citrate ligands. However, for successful embedding of gold nanoparticles in PEDOT:PSS, the nanoparticles are sterically stabilised with PEG (polyethylene glycol) instead of citrate. We use a protocol according to literature [9] for the PEG functionalisation of gold nanoparticles starting with the citrate stabilized nanoparticles from the Turkevich synthesis described above. The completeness of the encapsulation and successful stabilization by PEG has been verified for various PEG concentrations by absorbance spectra measurements of the plasmon resonance peak at about $530 \mathrm{~nm}$ after one week.

\subsection{Optical, topography and electrical characterization}

Refractive index, extinction coefficients, and layer thickness are measured using optical white light interferometry (Filmetrics). Optical transmission spectra of the deposited layers are recorded using an UV-visNIR spectrometer (Oceanoptics USB2000+). The overall distribution of $\mathrm{Au}$ nanoparticles within thin nanocomposite layers is investigated using a reversed microscope (Leica DMILM). Nano- and microstructures of Au-PEDOT:PSS nanocomposite printed features are analyzed by atomic force microscopy (AFM, Park) topography and phase contrast images, as well as optical 3D profilometry (Filmetrics). For estimating the electrical conductivity of thin films, we use a four-point collinear probing head with a lateral tip spacing of $1 \mathrm{~mm}$ together with a Keithley 2400 source meter. The probe head is positioned using a home-made set-up with a micro screw for comparable measurement conditions inspite of mechanical contact and possible layer damage [6].

\section{RESULTS AND DISCUSSION}

Figure 1 shows the optical absorbance spectra (UV-vis-NIR) of synthesized and commercial gold nanoparticle dispersions with citrate and PEG stabilisation, respectively. The spectra with an absorbance peak at about $530 \mathrm{~nm}$ confirm the main nanoparticle size of approximately $40 \mathrm{~nm}$ [9] in agreement with the data sheet indications for the commercial nanoparticles.

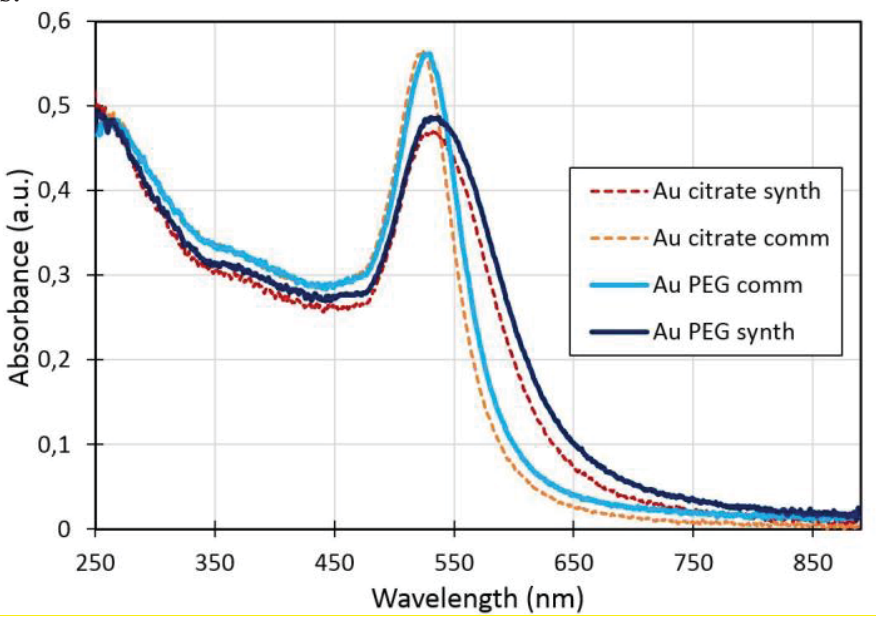

Figure 1. Absorption spectra (UV-vis-NIR) of gold nanoparticle dispersions (synthesized and commercial ones): citrate stabilized Au nanoparticles (dashed lines) and PEG stabilized Au nanoparticles (solid lines).

\subsection{Inkjet printed embedded Au nanoparticles}

Figure 2 shows representative AFM data observed on inkjet printed PEDOT:PSS with embedded gold nanoparticles. The observable protrusions (Fig 2a,b) have been found to have a height of up to approximately 10 $\mathrm{nm}$ with respect to their surroundings. We observe that their density of appearance increases with increasing additive load of gold nanoparticles (not shown here) and thus attribute them to embedded Au nanoparticles. We note however, that the inherent roughness and domain structure of PEDOT:PSS exhibits domain sizes similar to 
the nanoparticle diameter, which does not allow for a systematic identification of the $40 \mathrm{~nm}$ Au NPs from the AFM topography data of the printed structures.
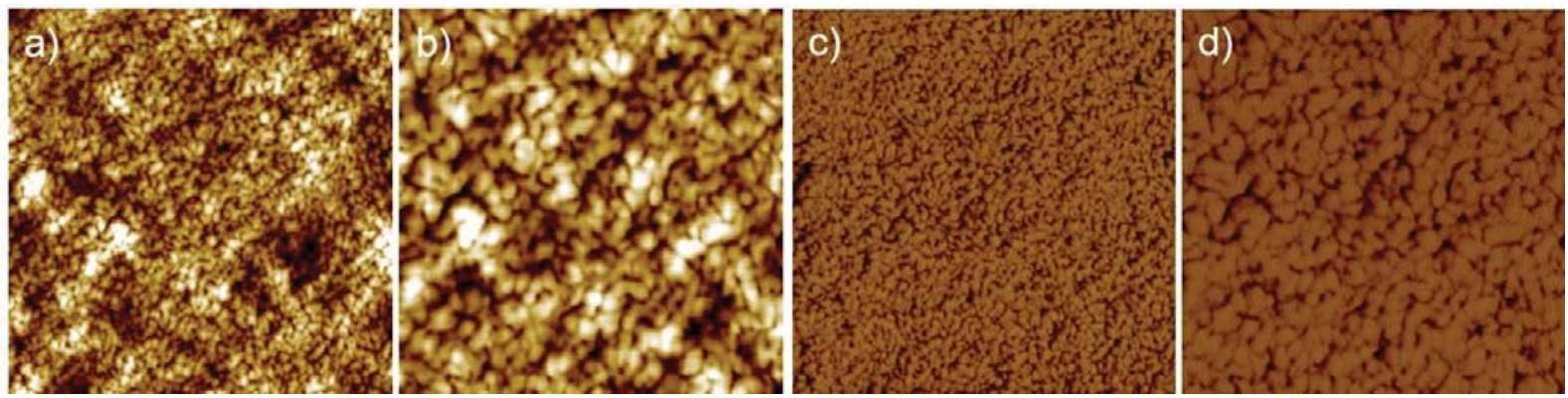

Figure 2. AFM topography images $(a, b)$ and their phase contrast images $(c, d)$ upon inkjet printing of the composite PEDOT:PSS-AuNP ink (scan size: a) and c) $2 \mu \mathrm{m} \times 2 \mu \mathrm{m}, b$ ),d) $1 \mu \mathrm{m} \times 1 \mu \mathrm{m}$.

The observed lack of particular contrast in AFM phase contrast images (e.g. Fig. c,d) is nevertheless well in agreement with successfully embedded nanoparticles.

\subsection{Nano- and microstructure features from inkjet printing of gold nanoparticles on top of PEDOT:PSS}

Figure 3 shows AFM topography data of Au nanoparticles that were deposited by inkjet printing on top of an inkjet printed PEDOT:PSS layer. In this case nanoparticles can be clearly identified from the AFM topography data and can be well distinguished from the PEDOT:PSS domain structure (Fig- 3b,c).
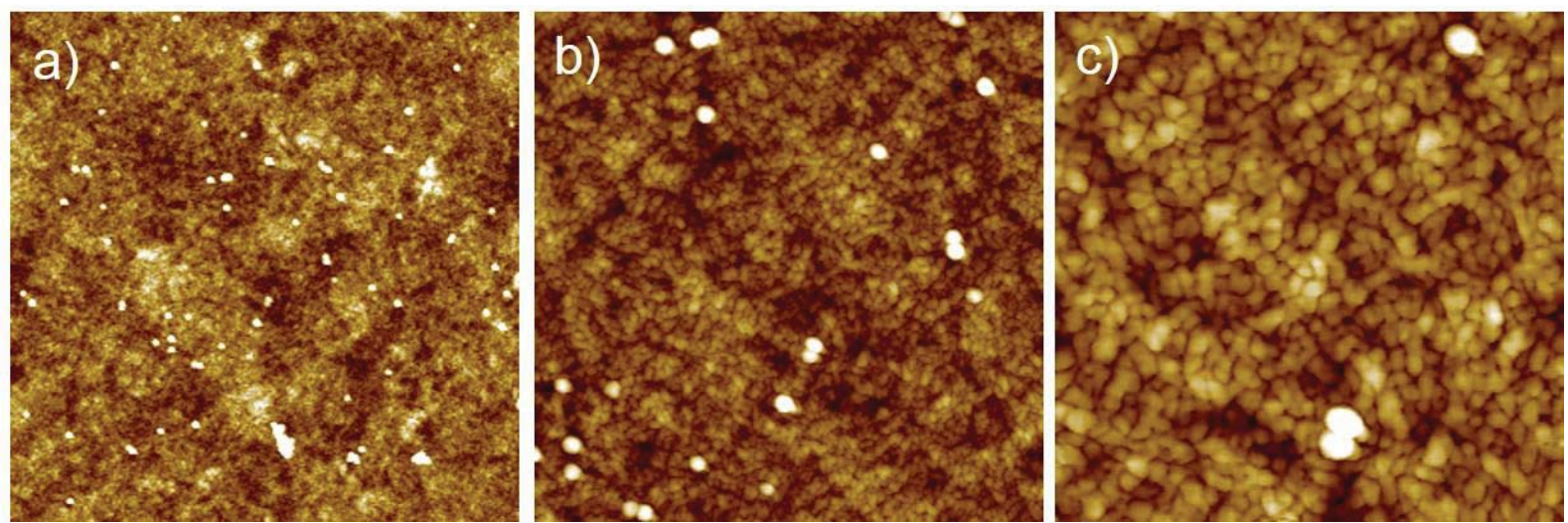

Figure 3. AFM topography images of Au NPs (commercial, PEG stabilized, centrifugated) upon printing on printed PEDOT:PSS (scan size: a) $5 \mu m \times 5 \mu m$, b) $2 \mu m \times 2 \mu m$, c) $1 \mu m \times 1 \mu m$.

Figure 4 shows 3D profilometer measurements of inkjet printed nanoparticle patterns printed on PEDOT:PSS printed lines. The nanoparticles are clearly identified and reveal sharp structures in optical profilometer data.
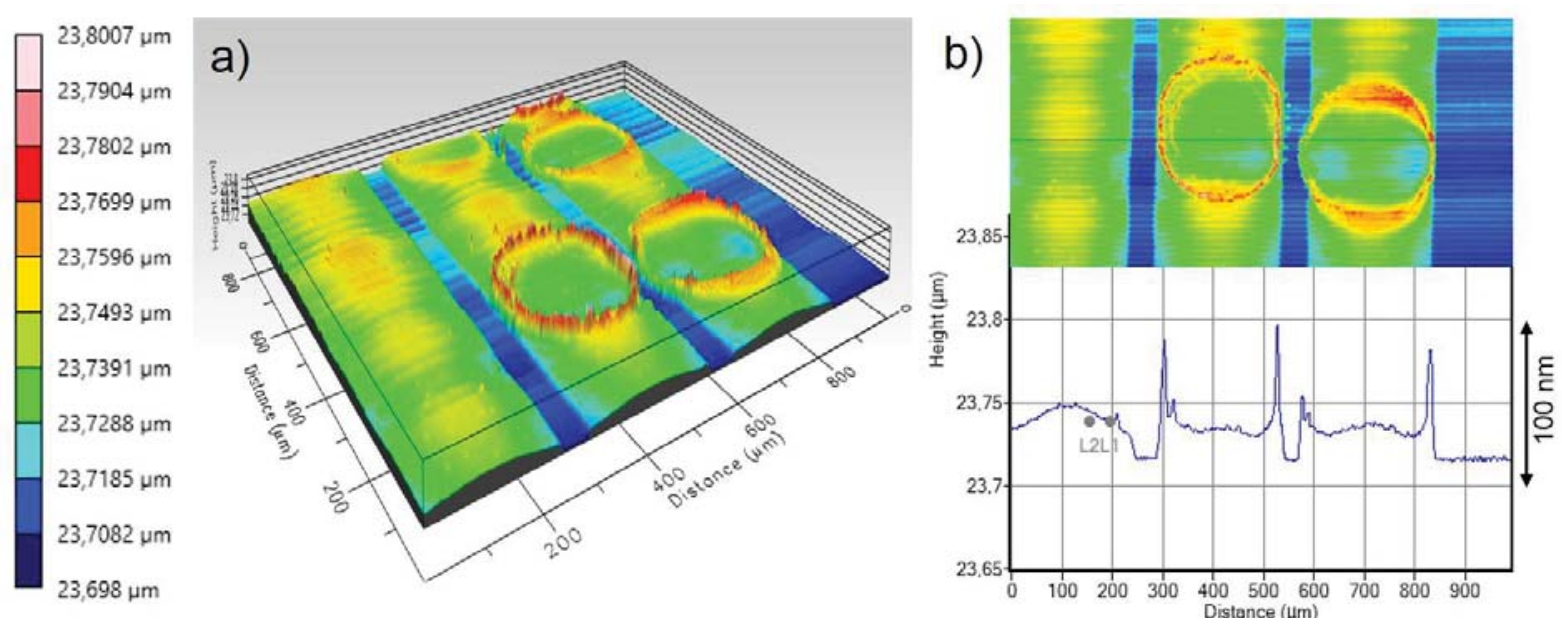

Figure 4. Inkjet printed PEDOT:PSS and Au nanoparticles, 3D optical profilometer measurements: a) 3D view $(1 \mathrm{~mm} \times 1 \mathrm{~mm}), b)$ top view and example of a line profile. Nanoparticles stay on top of PEDOT:PSS, their apparent height is in agreement with their diameters. 
From the line profiles, e.g. Fig 4b, the observed gold nanoparticles heights above the printed PEDOT:PSS line are essentially consistent with their nominal diameter. The observed height of the printed PEDOT:PSS is about $25 \mathrm{~nm}$, which is consistent with the observed height difference of about $10 \mathrm{~nm}$ for embedded nanoparticles with their surroundings (Fig.2). The circular structure observed for the printed nanoparticles is due to the coffee stain effect, which we propose here as a tool for the fabrication of plasmonic nano- and microstructures.

\section{CONCLUSIONS}

We have studied plasmonic gold nanoparticle PEDOT:PSS composites for inkjet printing applications. Gold nanoparticles functionalized with PEG were successfully embedded in PEDOT:PSS. Successive printing of PEDOT:PSS and gold nanoparticles leads to microstructure patterns that are electrically conductive and simultaneously contain optical nano- and microstructured features. Structuring has here been obtained using inkjet printing while making use of the coffee stain effect that leads to nanoparticle assembly at the circumference of the printed droplets. We propose this technique for further developments of smart security tags that have several physical features at once, such as here conducting properties of the PEDOT:PSS, as well as optical features by the plasmonic nanoparticles.

\section{ACKNOWLEDGEMENTS}

This work has been financially supported by HEIG-VD and HES-SO, project NanoInk (grant number 0222015 PI). We kindly acknowledge financial support by the SEMP (Swiss European Mobility Program) having granted a student fellowship for D. Palma de Barros. We like to acknowledge Hongyan Bi, for her scientific and experimental guidance and scientific discussions having strongly contributed to the implementation of $\mathrm{Au}$ nanoparticle synthesis to our laboratory.

\section{REFERENCES}

[1] Valerio Zardetto, Thomas M. Brown, Andrea Reale, and Aldo Di Carlo, Substrates for Flexible Electronics: A Practical Investigation on the Electrical, Film Flexibility, Optical, Temperature, and Solvent Resistance Properties, Journal of Polymer Science Part B: Polymer Physics 49, 638-648 (2011).

[2] John Turkevich. Colloidal gold. Part.II, Gold Bulletin 18(4), 125-131, 1985.

[3] Stephan Kirchmeyer and Knud Reuter, Scientific importance, properties and growing applications of poly(3,4-ethylenedioxythiophene), J. Mater. Chem. 15, 2077-2088 (2005)

[4] Manrico Fabretto, Kamil Zuber, Carlos Jariego-Moncuncill, Peter Murphy, Measurement Protocols for Reporting PEDOT Thin Film Conductivity and Optical Transmission: A Critical Survey, Macromolecular Chemistry and Physics 212, 2173 (2011)

[5] Ziyang Hu, Jianjun Zhang, and Yuejin Zhu, Effects of solvent-treated PEDOT:PSS on organic photovoltaic devices, Renewable Energy 62, 100-105 (2014).

[6] Nikolay Kurtev, Narcis Fosso, Charles Tematio, Slavka Tzanova, and Silvia Schintke, Electrical characterization of transparent organic conducting thin films preventing mechanical damage and preserving optical transparency, Electron Devices and Solid-State Circuits, EDSSC 2015, IEEE International Conference on, pp. 341-344, doi: 10.1109/EDSSC.2015.7285120.

[7] C. Tematio, N. Fosso, J. Krähenbühl, and S. Schintke, Conductive polymer nanocomposites for transparent circuits and thin films, Transparent Optical Networks, ICTON 2016, 18th International Conference on, pp. 1-5, IEEE Conference Publication, DOI: 10.1109/ICTON.2016.7550272.

[8] John Turkevich, Colloidal gold. Part I, Gold Bulletin 18(3), 86-91, 1985.

[9] Joanne Manson, Dhiraj Kumar, Brian J. Meenan, and Sorian Dixon, Polyethylene glycol functionalized gold nanoparticles: the influence of capping density on stability in various media. Gold Bulletin 44, 99105 (2011). 